\title{
Extracorporeal Membrane Oxygenation (ECMO) Transpires to be a Promising Surrogate Therapy for Severe Refractory near Fatal Asthma: A Case Report
}

\author{
Ali Al Bshabshe, MD, FRCP ${ }^{*}$, Omprakash Palanivel', Mohammed Amer Bahis ${ }^{3}$, Nasser Mohammed \\ Alwadai, $R_{R} T^{4}$ and Ali Mushabebassiri ${ }^{4}$
}

${ }^{1}$ Department of Medicine/Critical Care, College of Medicine, King Khalid University, Saudi Arabia

${ }^{2}$ Cardio-Respiratory Specialist, Aseer Central Hospital, Saudi Arabia

${ }_{3}^{3}$ Internvist Consultant, Aseer Central Hospital, Saudi Arabia

${ }^{4}$ Respiratory Therapist, Aseer Central Hospital, Saudi Arabia

*Corresponding author: Ali Al Bshabshe, MD, FRCP, Associate Professor of Medicine/Critical Care, College of Medicine, King Khalid University, Abha, Saudi Arabia

\begin{abstract}
The standard first-line therapy for severe asthma exacerbation is bronchodilators and corticosteroids. Any patients not responding to this standard therapy often needs invasive ventilation, which can promote numerous related complications and mortality. Recent studies conclude that ECMO can be adopted as an alternative approach as it significantly enhances the survival rate in severe respiratory failure compared to standard invasive ventilation. In contrast, ECMO effectiveness in near-fatal asthma is not well studied, possibly due to unpredictable asthma death before reaching the hospital and lack of current evidence-based studies that support the usage and benefits of ECMO. Herein, we present 39-year-old male as a case of near-fatal asthma with severe persistent hypercapnic respiratory failure refractory to conventional ventilation. Our early application of VV-ECMO for continuous 58 hours improved the patient hemodynamics and respiratory mechanics along with a rapid reduction of carbon dioxide tension. Thus it reduces the further complications and the length of the intensive care unit. Our case demonstrates the significance of the early hours of ECMO as promising surrogate therapy when conventional treatment fails. Long-term research urged to validate $\mathrm{VV}$ ECMO effectiveness in this group of patients.
\end{abstract}

\section{Keywords}

ECMO: Extracorporeal membrane oxygenation, NFA: Near fatal asthma, ABG: Arterial blood gas

\section{Introduction}

Asthma is a chronic inflammatory disorder of the airways associated with variable expiratory flow, airway wall thickening, respiratory symptoms, and exacerbations (flare-ups), possibly require hospitalizations and may be fatal [1]. It is the most prevalent chronic global disorder with an incidence of 235 million people in the world community [2]. Despite the succession of modern pharmacological therapy in asthma, hospitalization for asthma exacerbation is significant until the present, and mortality after admission to critical care is increasing $[3,4]$. The near fatal asthma (NFA) is a life-threatening condition of asthma associated with characteristics of hypoxemia, hypercapnia, altered mental status, not responding to conventional therapy, and often requiring mechanical ventilation. Mechanical ventilation can generate harmful outcomes in NFA due to the presence of worsening dynamic hyperinflation and increase intrathoracic pressure, which may result in hemodynamic instability and sudden cardiovascular arrest. The reported mortality rate in patients who are in ventilator assistance is $30 \%$. Also, Mechanical ventilation might lead to several complications attributed to the related mortality of 7-8\% [5]. The ECMO is an alter-

Citation: Al Bshabshe A, Palanivel O, Bahis MA, Alwadai NM, Mushabebassiri A (2020) Extracorporeal Membrane Oxygenation (ECMO) Transpires to be a Promising Surrogate Therapy for Severe Refractory near Fatal Asthma: A Case Report. Int J Crit Care Emerg Med 6:112. doi.org/10.23937/24743674/1510112

Accepted: December 01, 2020: Published: December 03, 2020

Copyright: (C) 2020 Al Bshabshe A, et al. This is an open-access article distributed under the terms of the Creative Commons Attribution License, which permits unrestricted use, distribution, and reproduction in any medium, provided the original author and source are credited. 


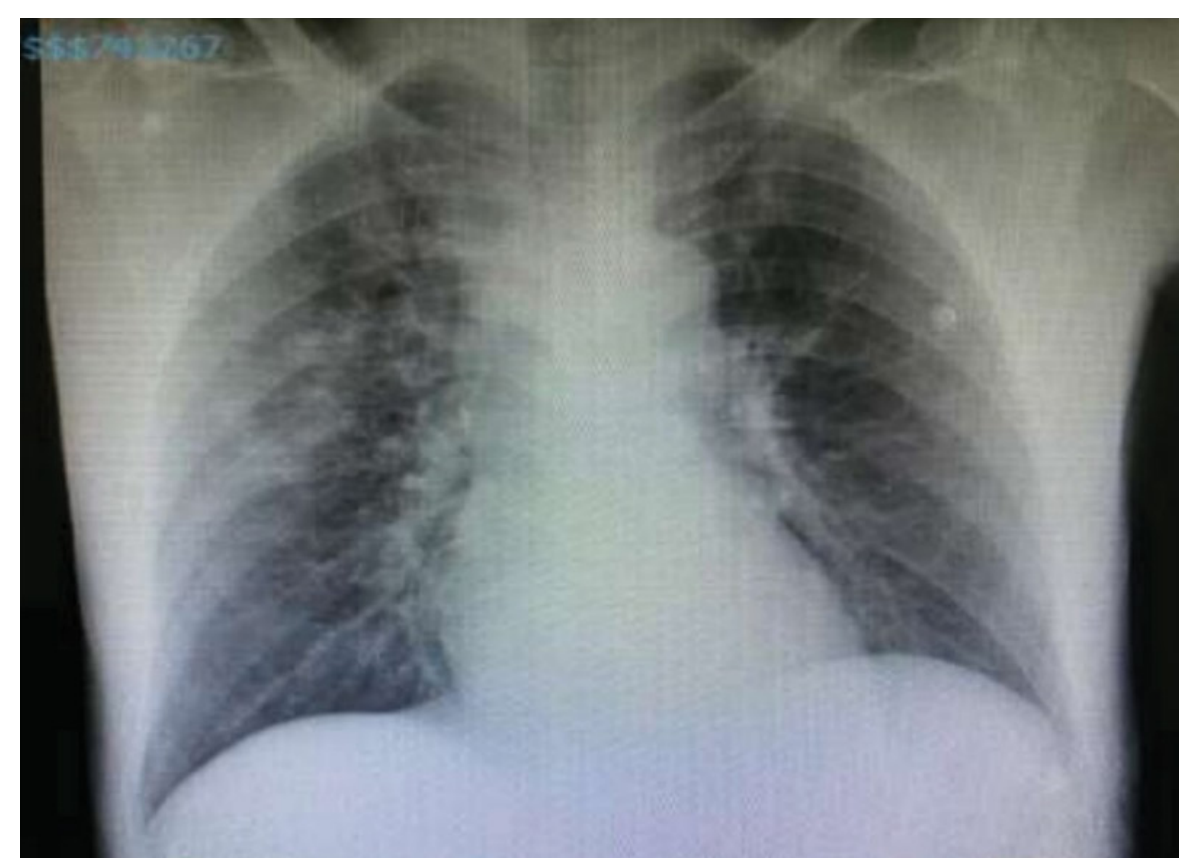

Figure 1: X-ray admission.

native approach of cardio-pulmonary support in which oxygen supplemented and carbon dioxide $\left(\mathrm{CO}_{2}\right)$ is expelled through an extracorporeal membrane using arteriovenous or venovenous cannulation. The goal of this therapy is to minimize ventilator-induced lung injury and to provide adequate time for the lung to heal from the inflammatory process. Several present studies conclude that ECMO enhances survival in adults with reversible respiratory failure compared to mechanical ventilation. Although NFA usually occurs in healthy individuals, and it is potentially reversible, its benefits from ECMO is limited and confined to a few case reports $[6,7]$. We present a case of successful early use of ECMO for 58 hours to correct the persistent severe hypercapnic reversible respiratory failure that was refractory to conventional ventilation.

\section{Case Report}

A 39-year-old male known smoker history of childhood bronchial asthma on irregular asthmatic medications was reported to the emergency room (ER) with acute respiratory distress and shortness of breath refractory to inhaled SABA and productive cough for one day. The past clinical history summarized one episode of emergency admission to the hospital before 14 months with similar clinical symptoms and responded well to standard first-line therapy without the need for noninvasive or invasive ventilation. Presently, on examination, the patient is in severe respiratory exhaustion using accessory muscles, his vitals are blood pressure $145 / 84 \mathrm{mmHg}$, regular heart rate $125 / \mathrm{min}$, respiratory rate $43 / \mathrm{min}$, temperature $37^{\circ} \mathrm{C}$, oxygen saturation was $84 \%$ on room air. His Physical examination: Weight of $82 \mathrm{~kg}$, height of $174 \mathrm{~cm}, \mathrm{BMI} 28 \mathrm{~kg} / \mathrm{m}^{2}$, mild jugular distension, Glasgow Coma Scale (GCS) score was 15/15. On auscultation, diffuse wheezing with insignificant bi- lateral breath sounds. Anteroposterior chest X-ray revealed bilateral increased Bronchovascular markings, and hyperinflated lungs (Figure 1) and electrocardiogram showed sinus tachycardia. In an emergency room, the patient treated with $\mathrm{O}_{2} 15 \mathrm{~L} / \mathrm{min}$ via a non-breathing mask, and the oxygen saturation rose to $90 \%-91 \%$. His initial blood biochemistry was within normal limits. His treatment in the emergency room included intravenous fluids of normal saline $500 \mathrm{ml}$, hydrocortisone $100 \mathrm{mg}$ TID, methylprednisolone $125 \mathrm{mg}$ intravenous stat, 2 gram of magnesium sulphate, continuous inhaled ipratropium bromide, inhaled salbutamol. Arterial blood gas analysis (ABG) obtained, nasal swab for merscov and sputum for cultures collected. His initial ABG showed respiratory acidosis $\mathrm{Ph} 7.23 \mathrm{pCO}_{2} 75.3$ pO 72.9 $\mathrm{HCO}_{3} 24.6 \mathrm{mmol} / \mathrm{L}$. The diagnosis at admission was severe acute asthma exacerbation. His condition deteriorated subsequently with desaturation to $79 \%$ with $15 \mathrm{~L} /$ min of oxygen. A trial of noninvasive ventilation (NIV) via BIPAP was initiated to reduce the work of breathing to promote oxygenation and ventilation. Nevertheless, he cannot endure the inspiratory pressure of $10 \mathrm{cmH}_{2} \mathrm{O}$ and expiratory pressure of $4 \mathrm{cmH}_{2} \mathrm{O}$. Repeated ABG revealed increasing respiratory acidosis: $\mathrm{pH} 7.14, \mathrm{pCO}_{2}$ $96.7 \mathrm{mmHg}, \mathrm{pO}_{2} 63 \mathrm{mmHg}, \mathrm{HCO}_{3} 23.4 \mathrm{mmol} /$, and low GCS (11/15). Therefore, he transferred to the intensive care unit (ICU) for close observation and he continued to deteriorate over the next 60 minutes from admission with an altered mental status (GCS 9/15) and hypopnea with worsening respiratory acidosis: $\mathrm{pH} 7.09$, $\mathrm{pCO}_{2} 106 \mathrm{pO}_{2} 61 \mathrm{HCO}_{3}$ 21.4. He was electively intubated in a rapid sequence manner, and post-intubations $X$-ray illustrated hyperinflated lungs. Started on volume control mode ventilation with $\mathrm{FiO}_{2}$ of 1.0 , respiratory rate of 18 , tidal volume $5 \mathrm{ml} / \mathrm{kg}$, I:E ratio of 1:4, positive end-expiratory pressure (PEEP) $3 \mathrm{cmH}_{2} \mathrm{O}$. Furthermore, 
to bridge synchrony between the patient and ventilator. The patient was well sedated with propofol $70 \mathrm{mg} / \mathrm{hr}$, midazolam $7 \mathrm{mg} / \mathrm{hr}$, fentanyl $100 \mathrm{mcg} / \mathrm{hr}$, ketamine 50 $\mathrm{mg} / \mathrm{hr}$, atracurium $40 \mathrm{mg} / \mathrm{hr}$. In addition, high dose of inhaled bronchodilators (salbutamol $5 \mathrm{mg} / \mathrm{hr}$, adrenalin $1 \mathrm{mg} / \mathrm{hr}$, ipratropium $0.5 \mathrm{mg} / \mathrm{hr}$ ), intravenous corticosteroid (methylprednisolone $80 \mathrm{mg} / 8 \mathrm{~h}$ ) and magnesium sulfate $12 \mathrm{~g} / 24 \mathrm{~h}$ were included. Despite standard measures, peak airway and plateau pressure are at 57 and $40 \mathrm{cmH}_{2} \mathrm{O}$, dynamic compliance is $13 \mathrm{ml} / \mathrm{cmH}_{2} \mathrm{O}$, and total PEEP of $18 \mathrm{cmH}_{2} \mathrm{O}$. His continuous air trapping, high Peak, and high plateau pressure are in the high chance to promote barotrauma. Hence, the tidal volume, peep are reduced to $3.0 \mathrm{ml} / \mathrm{kg}$ and $0 \mathrm{cmH}_{2} \mathrm{O}$, respectively. Despite adequate manipulation of mechanical ventilation, the outcome of respiratory mechanics is unsatisfactory. Therefore, pressure control ventilation was initiated at $6^{\text {th }}$ hour of post-intubation with $\mathrm{FiO}_{2} 1.0, \mathrm{RR} 18, \mathrm{PEEP}$ $0, P C$ 30. Thereby Peak alveolar pressure and the risk of barotrauma were minimized. However, the hypercapnic respiratory failure persisted in the patient's serials of blood gases with no improvements along with high plateau pressure $>37 \mathrm{cmH}_{2} \mathrm{O}$. The arterial blood gas values at $8^{\text {th }}$ hours of post-intubation were: $\mathrm{pH} 7.06$, $\mathrm{pCO}_{2} 111 \mathrm{mmHg}, \mathrm{PO}_{2} 63 \mathrm{mmHg}, \mathrm{HCO}_{3}-20 \mathrm{mmol} / \mathrm{L}$. At this moment, our team decides to initiate veno-venous extracorporeal membranous oxygenation (VV ECMO) based on the following clinical presentation; $\mathrm{paO}_{2} / \mathrm{FiO}_{2}$ which remained $<100 \mathrm{mmHg}$ after 10 hours of mechanical ventilation, respiratory acidosis with a $\mathrm{pH}<7.2$, high plateau pressure $\geq 35 \mathrm{~m}$ bar with tidal volumes $>3.5 \mathrm{ml} /$ kg ideal body weight (IBW), invasive ventilation for less than 3 days, Murray lung injury score $\geq 3$, and to prevent the risk of ventilation-induced lung injury.
VV-ECMO Cannulation is performed with complete sterile measures of seldinger technique under the guidance of ultrasonography. A draining cannula of $25 \mathrm{fr}$ is inserted into the right femoral vein and advanced into the inferior vena cava. Likewise, a returning cannula of $19 \mathrm{fr}$ is inserted into the right internal jugular vein and advanced into the superior vena cava (Figure 2). A centrifugal pump used was Getinge/Maquet ${ }^{\circ}$, Rastatt, Germany, with an initial flow of $2 \mathrm{~L} / \mathrm{min}$, sweep gas $2 \mathrm{~L} / \mathrm{m}$. Heparin $5000 \mathrm{IU}$ at cannulation. Then $1200 \mathrm{IU}$ per hour to keep the target APTT ratio of 1.5-2.5 Immediate after ECMO cannulation, the blood gas value rapidly normalized. The first set of BGA Values at 60 minutes after starting ECMO were: $\mathrm{pH} 7.143, \mathrm{pCO}_{2} 86.2 \mathrm{mmHg}, \mathrm{pO}_{2} 74.9$ $\mathrm{mmHg}, \mathrm{HCO}_{3}^{-} 21.5 \mathrm{mmol} / \mathrm{L}$. There was no complication associated with VV ECMO cannulation. Adequate gas exchanges achieved with pressure control ventilation at PIP of $22 \mathrm{cmH}_{2} \mathrm{O}$, rate 12/min, $\mathrm{FiO}_{2} 40 \%$, and PEEP $3 \mathrm{cmH}_{2} \mathrm{O}$ and decide to maintain the same MV settings for next 60 hours in adjunct with VV ECMO. The patient monitored closely with an hourly recording of all vitals; ABGs were repeated at every 2-hour interval for first 24 hours and every $6^{\text {th }}$ hourly for the next 34 hours of ECMO therapy.

After 58 hours of continuous ECMO, the patient's clinical condition improved significantly, along with a reduction in $\mathrm{PCO}_{2}$ to $39.6 \mathrm{mmHg}$. Meanwhile, during 58 hours of ECMO, the patient was kept well sedated and ventilated; he continued on antibiotics, nebulization, steroids, and IV salbutamol, heparin infusion along with atracurium $40 \mathrm{mg}$ per hour. By day 3, ECMO was weaned off by gradual flow and discontinued without any associated complications (Figure 3). On day 4, he was on a spontaneous breathing trial, respiratory mechani-

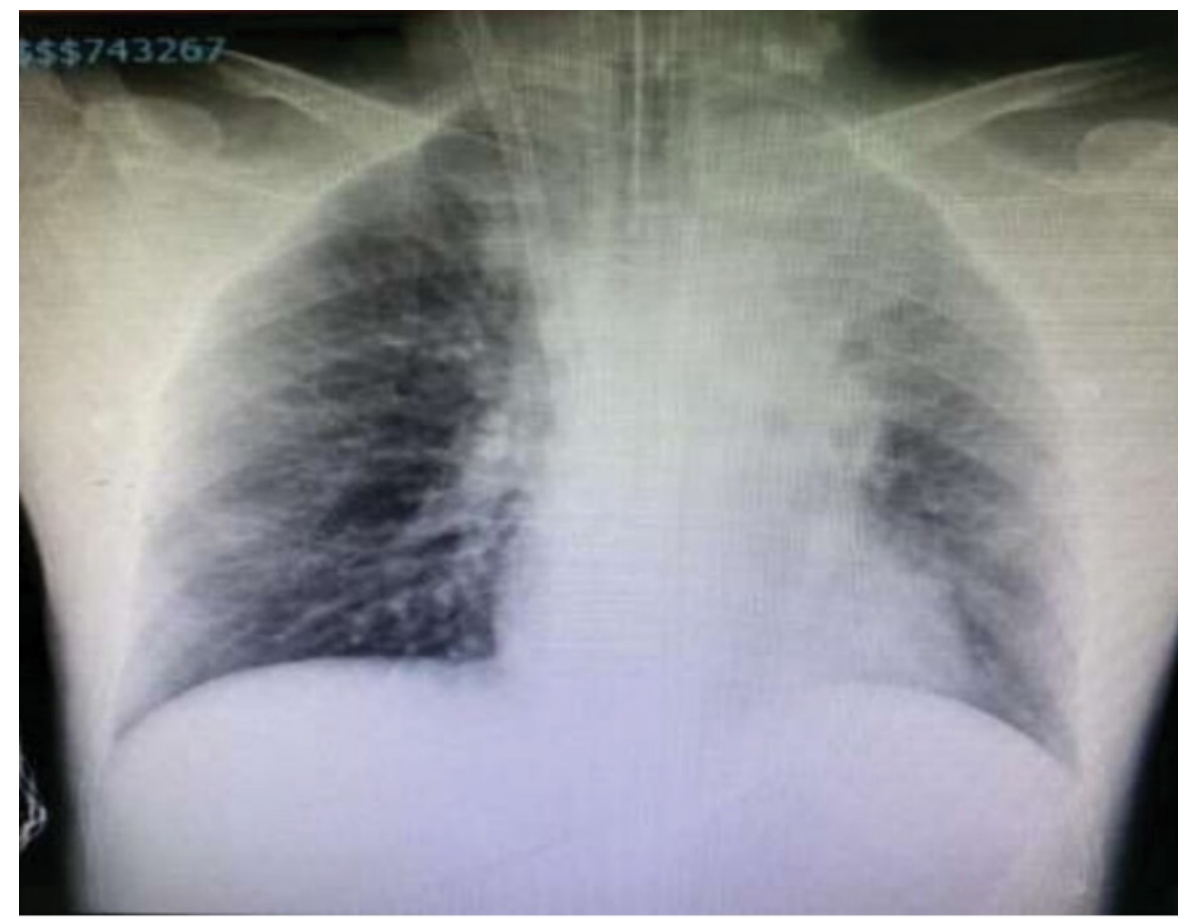

Figure 2: X-ray immediate after VV-ECMO cannulation. 


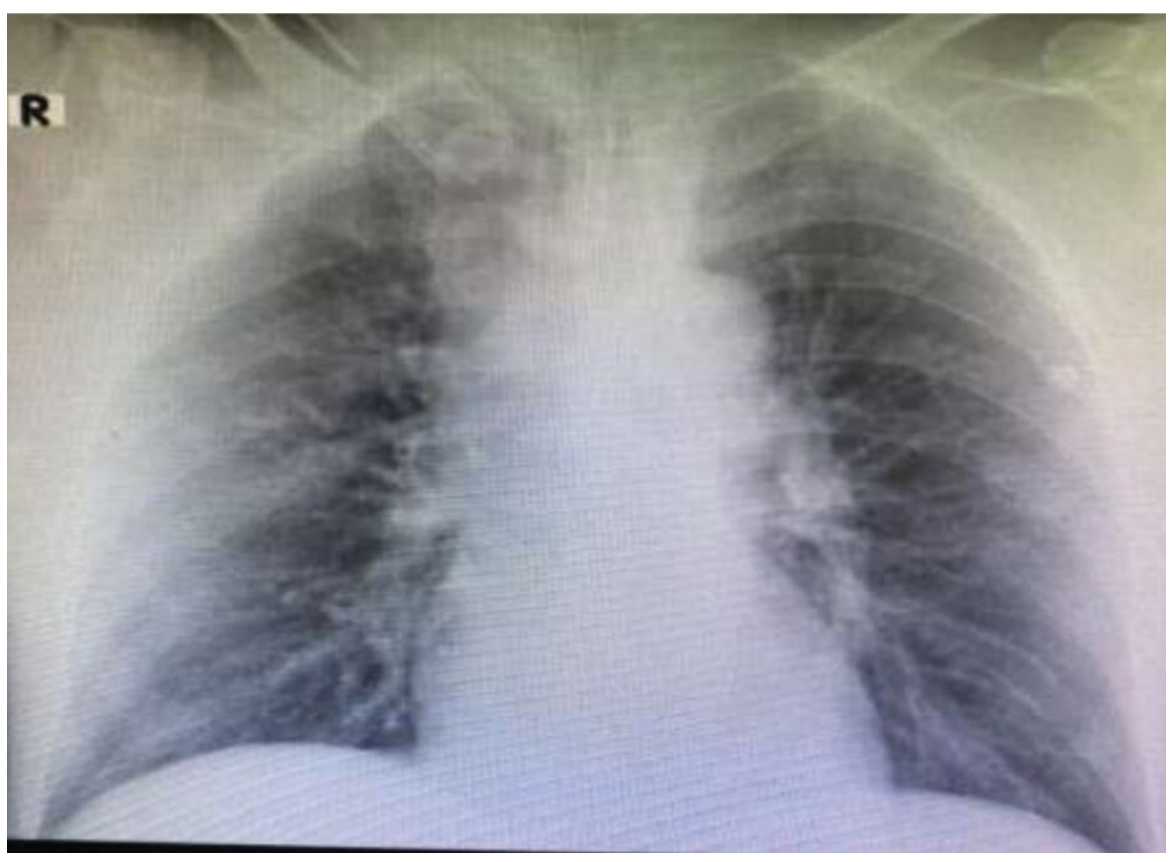

Figure 3: X-ray after decannulation of VV-ECMO.

Table 1: Blood gas parameters before ECMO and during and after ECMO.

\begin{tabular}{|c|c|c|c|c|c|c|c|c|c|c|c|c|c|c|c|c|}
\hline \multicolumn{17}{|c|}{ Blood gas parameters before ECMO and during and after ECMO } \\
\hline \multirow{3}{*}{$\begin{array}{l}\text { Parameters } \\
\mathrm{pH}\end{array}$} & \multirow{2}{*}{\multicolumn{3}{|c|}{ Before ECMO }} & \multirow[b]{3}{*}{7.06} & \multicolumn{11}{|c|}{ ECMO 58 HOURS } & \multirow{3}{*}{$\begin{array}{l}\text { After ECMO } \\
7.47 \\
\end{array}$} \\
\hline & & & & & \multirow{2}{*}{\begin{tabular}{|l}
$2^{\text {nd }}$ \\
7.13
\end{tabular}} & \multirow{2}{*}{\begin{tabular}{|l|}
$2^{\text {th }}$ \\
7.18 \\
\end{tabular}} & \multirow{2}{*}{\begin{tabular}{|l|}
$10^{\text {th }}$ \\
7.21
\end{tabular}} & \multirow{2}{*}{$\begin{array}{l}16^{\text {th }} \\
7.25\end{array}$} & \multirow{2}{*}{\begin{tabular}{|l}
$22^{\text {th }}$ \\
7.25
\end{tabular}} & \multirow{2}{*}{\begin{tabular}{|l|}
$28^{\text {th }}$ \\
7.27
\end{tabular}} & \multirow{2}{*}{\begin{tabular}{|l}
$34^{\text {th }}$ \\
7.37
\end{tabular}} & \multirow{2}{*}{$\begin{array}{l}40^{\text {nd }} \\
7.41\end{array}$} & \multirow{2}{*}{$\begin{array}{l}46^{\text {th }} \\
7.41\end{array}$} & \multirow{2}{*}{\begin{tabular}{|l}
$52^{\text {th }}$ \\
7.41
\end{tabular}} & \multirow{2}{*}{$\begin{array}{l}58^{\text {th }} \\
7.46\end{array}$} & \\
\hline & 7.23 & 7.14 & 7.097 & & & & & & & & & & & & & \\
\hline $\mathrm{pCO}_{2}$ & 75.3 & 96.7 & 106 & 111 & 70.6 & 68.1 & 62.9 & 61.8 & 58.0 & 56.9 & 49.7 & 45.1 & 41.7 & 40.1 & 39.6 & 34.2 \\
\hline $\mathrm{pO}_{2}$ & 72.9 & 52.2 & 61.2 & 63 & 98.7 & 64.8 & 65.1 & 61.9 & 63.2 & 64.9 & 59.0 & 70.4 & 75.0 & 85.9 & 82.1 & 108 \\
\hline $\mathrm{HCO}_{3}$ & 24.6 & 23.4 & 21.4 & 22.5 & 19.0 & 20.6 & 20.5 & 23.0 & 22.0 & 23.1 & 26.5 & 27.7 & 25.8 & 25.4 & 26.6 & 26 \\
\hline $\mathrm{SO}_{2}$ & 92.1 & 80.5 & 97.2 & 92.2 & 97 & 98.1 & 90.4 & 92.5 & 91.2 & 91.2 & 93.1 & 96.4 & 95.2 & 97 & 98.6 & 98.6 \\
\hline
\end{tabular}

$\mathrm{Cs}$, and $\mathrm{ABG}$ is ph $7.47 \mathrm{pCO}_{2} 34.2 \mathrm{pO}_{2} 108 \mathrm{HCO}_{3} 26$ satisfactory. Therefore, he was extubated and transferred to the ward on day 6 and discharged home on day $16^{\text {th }}$ without any complications. The serials of high ligtened $\mathrm{PCO}_{2}$ reduction in over 58 hours of ECMO therapy are shown in the Table 1. He turned to be influenzas B infection from nasal swab result exacerbating his near fatal asthma episode and it has been treated with Tamiflu $75 \mathrm{mg}$ BID for 5 days.

\section{Discussion}

The key factors that differentiate the patient of near-fatal asthma from severe asthma are: If there is no response to repeated sessions of Beta 2-agonist therapy, which results in respiratory failure necessitating hypercapnia (> $6.5 \mathrm{kPa}$ ), with or without altered consciousness for ICU admission for the possible requirement of mechanical ventilation, or history of the previous hospitalization [8]. The MV in NFA patients is a lifesaving emergency act as well; it requires a high dosage of sedation, analgesics, and muscle relaxants for good MV synchrony. At the same time, high sedation can increase further bronchospasms, air trapping, hyperinflation, which results in low functional residual capacity and in- creased intrathoracic pressures affecting the gas exchange $[9,10]$. While recent days asthma mortality is declining but remains significant. Substantial mortality and morbidity seen in asthma may be associated with MV itself in comparison to disease progression. In this case, our main objective is to reduce alveolar hyperinflation, avoid barotrauma, and maintain adequate oxygenation and ventilation by acknowledging some degree of permissive hypercapnia, until bronchodilators and steroids reverse the severe airflow resistance [11]. Nevertheless, with all these high sedation and ultra-protective lung strategy, the ventilator waveform of our patient exhibits persistent high peak airway pressure, high plateau pressure, and air trapping, respectively. To prevent these possible harmful effects of ventilator-induced lung injuries Extracorporeal Membrane Oxygenation (ECMO) adopted as surrogate therapy. Traditionally, ECMO commonly used in pediatrics with acute reversible respiratory and circulatory failure. Although the first case rescued successfully by ECMO in the year 1981. Still, ECMO remains controversial in these clinical settings. However, two large retrospective studies demonstrated the effectiveness and safety of ECMO in severe asthma attacks. In recent days ECMO plays a significant role in 
adult respiratory failure who failed in response to conventional treatments. Hyeju yeo, et al. concluded that among 24,147 patients 568 (2.4\%) were asthmatics of these, 272 patients were treated with ECMO. In whom rate of ECMO weaning success was 234/272 (86.70\%) and rate of survival to hospital discharge was 227/272 (83.5\%) in the multicenter International Extracorporeal Life Support Organization registry between march 1992 to march 2016 [12]. ECMO's success in asthma is due to the natural reversibility of the airflow obstruction, whi$c h$ is in contrast to the patient with diffuse alveolar damage due to ALI. In a recent report of acute respiratory failure due to the influenza A (H1N1) epidemic in 2009, applying ECMO to these patients was associated with favorable results [13]. Although the first case rescued successfully by ECMO in the year 1981. Still, ECMO remains controversial in the clinical setting. However, Qingyuan Zhan in his literature review recommended Algorithmic approach to initialize ECMO if $\mathrm{pH}$ is less than 7.1, $\mathrm{PaCO}_{2}$ greater than $100 \mathrm{mmHg}$, or any life-threatening conditions such as hypoxemia, hypercapnia, hypotension, or barotrauma and optimized ventilatory support. Correspondingly, our patient fit into these criteria and returned to normal respiratory function within 70 hours from ECMO initiation, particularly driving pressure, air trapping, and PIP, which significantly improved within a few hours of VV-ECMO running. Driving pressure is a transpulmonary pressure. Any high transpulmonary pressure can cause lung injury or barotrauma. The high PIP seen before ECMO initiation may reflect possible dynamic hyperinflation, barotrauma, cardiovascular instability, and even death $[14,15]$. Similarly, our patient prior to ECMO continues to be severe refractory hypercapnic respiratory failure remarkably on high ventilatory settings, which ascertained to destroy the lungs over time in association with toxic oxygen concentrations and increase pressures. Although, ECMO utilization does not play a vital role in healing the sick lungs; instead, it allows the lungs to rest while our patient's vital functions maintained safely. Besides, the ventilator settings can be adjusted down to very low settings until the lungs recover. Our report highlighted that early VV-ECMO could lower the PIP, driving pressure and air trapping rapidly, and it can improve the ventilation/perfusion mismatch significantly by decreasing airway resistance and airway obstruction. Also, by admitting low ventilator settings, VV-ECMO can overcome ventilator-induced lung injury and oxygen toxicity. Usually, the time course to reestablish airway obstruction by standard conventional treatment is profoundly variable, and physicians cannot determine when bronchospasm will resolve. Adoption of VV-ECMO as surrogate therapy in the early hours of severe hypoxemia, hypercapnia, and before generating any ventilator-associated lung injury is the key factor that influenced the survival of our patient.

\section{Conclusion}

In brief, Near-fatal asthma is a rare subclass of asth- ma and it's a life-threatening event. The early application of extracorporeal membrane oxygenation can influence the survival of the NFA. Our case signifies that VV ECMO should be utilized in the early hours of conventional treatment failure also before the occurrence of VILI, and even feasibly before the traits of any vital organ dysfunction. Notably, Physicians should be aware that each NFA patient may not be a candidate for ECMO. In context, ECMO can be adopted as surrogate therapy when the highest conventional treatment fails. A randomized controlled trial on the use of ECMO highly recommended in this group of patients.

\section{References}

1. Global initiative for asthma (GINA) (2019) Global Strategy for Asthma Management and prevention.

2. http://www9.who.int/respiratory/asthma/en/

3. Krishnan V, Diette GB, Rand CS, Bilderback AL, Merriman $B$, et al. (2006) Mortality in patients hospitalized for asthma exacerbations in the United States. Am J Respir Crit Care Med 174: 633-638.

4. Watson L, Turk F, Rabe KF (2007) Burden of asthma in the hospital setting: An Australian analysis. Int $\mathrm{J}$ Clin Pract 61: 1884-1888.

5. Linna Huang, Qingyuan Zhan (2019) An algorithmic approach for and special characteristics of veno-venous extracorporeal life support in patients with severe asthma attack: A case report and review of the literature.

6. Chang CL, Yates DH (2011) Use of early extra-corporeal membrane oxygenation (ECMO) for severe refractory status asthmaticus. J Med Cases 2: 124-126.

7. Mikkelsen ME, Pugh ME, Hansen-Flaschen JH, Woo YJ, Sager JS (2007) Emergency extracorporeal life support for asphyxic status asthmaticus. Respir Care 52: 1525-1529.

8. Papiris S, Kotanidou A, Malagari K, Roussos C (2002) Clinical review: Severe asthma. Crit Care 6: 30-44.

9. Anzueto A, Frutos-Vivar F, Esteban A, Inmaculada Alía, Laurent Brochard, et al. (2004) Incidence, risk factors and outcome of barotrauma in mechanically ventilated patients. Intensive Care Medicine 30: 612-619.

10. Hodder R, Lougheed MD, FitzGerald JM, Brian H Rowe, Alan G Kaplan, et al. (2010) Management of acute asthma in adults in the emergency department: assisted ventilation. CMAJ 182: 265-272.

11. Darioli R, Perret C (1984) Mechanical controlled hypoventilation in status asthmatics. Am Rev Respir Dis 129: 385-387.

12. HyeJu Yeo, Dohyung Kim, Doosoo Jeon, Yun Seong Kim, Peter Rycus, et al. (2017) Extracorporeal membrane oxygenation for life-threatening asthma refractory to mechanical ventilation: Analysis of the extracorporeal life support organization registry. Crit Care 21: 297.

13. Nithya Menon, Carlos M Perez-Velez, Jennifer A Wheeler, Michael F Morris, Orazio L Amabile, et al. (2017) Extracorporeal membrane oxygenation in acute respiratory distress syndrome due to influenza A (H1N1) pdm09 pneumonia. A single-center experience during the 2013-2014 season. Rev Bras Ter Intensiva 29: 271-278.

14. Amato MB, Meade MO, Slutsky AS, Laurent Brochard, Eduardo LV Costa, et al. (2015) Driving pressure and survival in the acute respiratory distress syndrome. $\mathrm{N}$ Engl $\mathrm{J}$ Med 372: 747-755.

15. Loring SH, Malhotra A (2015) Driving pressure and respiratory mechanics in ARDS. N Engl J Med 372: 776-777. 